\title{
Educación física y deporte: saberes corporales, género y colonialidad
}

\author{
Physical Education and Sport: Bodily Knowledge, Gender and \\ Coloniality
}

\author{
Fabián Horacio Martins \\ fabian.martins@crub.uncoma.edu.ar \\ Universidad Nacional del Comahue, \\ Centro Regional Universitario Bariloche, Argentina
}

Recepción: 17 Junio 2020

Aprobación: 25 Septiembre 2020

Publicación: 09 Octubre 2020

Cita sugerida: Martins, F. H. (2020). Educación física y deporte: saberes corporales, género y colonialidad. Educación Física y Ciencia, 22(4), e152. https://doi.org/10.24215/23142561e152
Resumen: El artículo toma como punto de partida un estudio previo vinculado a la selección de saberes deportivos a enseñar, en el área de la educación física; en escuelas secundarias de San Carlos de Bariloche. Describe los principales componentes que configuran la elaboración de un nuevo proyecto de investigación, orientado al análisis de las prácticas deportivas de alcance comunitario con participación juvenil y la problematización de las trayectorias estudiantiles junto a los equipos docentes. Dicho proyecto se enmarca dentro del enfoque interseccional y los estudios de las relaciones de poder bajo la tríada de opresión “raza-clase-género". Tiene por objetivo analizar los mecanismos de división sexual y las características que presentan en la región; además de observar los componentes lúdicos. La propuesta de investigación de corte educativa colaborativa, pretende contribuir a la reflexividad desde un abordaje reflexivo y crítico acerca de los procesos de selección de saberes a enseñar. Dentro de un enfoque cualitativo de tipo descriptivo, se fijaron criterios para abarcar la heterogeneidad de la población en estudio, según la ubicación geográfica y el tipo de gestión. El mismo podrá complementarse con técnicas estandarizadas que responden al modelo cuantitativo según las necesidades del trabajo.

Palabras clave: Educación física, Deportes, Saberes corporales, Género, Colonialidad.

Abstract: According to the previous study, the processes of selection of sports knowledge to be taught at secondary schools in San Carlos de Bariloche, the teaching teams do not incorporate the knowledge which would be interesting to the student community in everyday life. In this sense, we propose to carry out an investigation of community sports body practices with youth participation, within a collaborative educational research aimed at contributing to the reflexivity of these processes. Taking into account the power disputes structured from the triad gender-race-class oppression, we will analyze their mechanisms of sexual division and the particular characteristics which they present in our region, along with their playful components. In this way, we intend to problematize the student's trajectories within the debates related to the knowledge to be taught, in order to contribute to the improvement of educational quality in school institutions. Within a descriptive qualitative approach, we set criteria to cover the heterogeneity of the study population, according to the geographic location and the type of management. It could be complemented with standardized techniques that respond to the quantitative model according to the needs of the fieldwork and the purposes of this study.

Keywords: Physical Education, Sport, Bodily knowledge, Gender, Coloniality.

\section{Introducción:}

En el presente artículo describimos los principales componentes que conforman el proyecto de investigación vinculado al análisis de 
las prácticas deportivas de alcance comunitario con participación juvenil en San Carlos de Bariloche. Con este análisis buscamos contribuir al desarrollo de la reflexividad sobre los procesos de selección de saberes del área de educación física en las escuelas secundarias rionegrinas (en adelante ESRN). ${ }^{1}$

El enfoque interseccional que hace hincapié en las disputas de poder establecidas en función de la tríada de opresión género-razaclase, posibilita interpelar dichas prácticas. Asimismo, la perspectiva de investigación educativa colaborativa aporta a la problematización de los saberes deportivos a enseñar, desde la construcción de narrativas y la reflexión sobre las experiencias educativas.

Los antecedentes directos se sitúan en los resultados del estudio que llevamos a cabo con respecto a los procesos de selección y tratamiento de los saberes a enseñar en el área de educación física de las ESRN de San Carlos de Bariloche ${ }^{2}$. Los aspectos que allí consideramos remiten a sus relaciones con las trayectorias, el diseño curricular de nivel medio de Río Negro y la sociabilidad institucional, así como también la incidencia del ausentismo en las clases. Dato este último que evidenciamos a partir de los discursos que atribuyen sus causas al contra-turno, a la utilización de espacios externos a la institución escolar y la escasa predisposición de las juventudes hacia las actividades que involucran al cuerpo y al movimiento. Producto de este análisis, la pregunta que nos interpela respecto de los condicionantes que involucran directamente a las tareas de enseñanza es la siguiente: ¿Qué lugar ocupan las prácticas deportivas de interés de las juventudes dentro de los procesos de selección de saberes llevados a cabo por los equipos docentes de educación física? (Martins, 2019).

Los resultados de la investigación exhiben un cierto desconocimiento hacia estas prácticas producto, entre otros factores, de las condiciones laborales de la comunidad docente y la tendencia a considerarlas desde su carácter universal. Se destaca así su papel formativo, más allá de las modalidades que asuma y los propósitos que persiga su enseñanza. Queda en evidencia que los procesos de selección de saberes a enseñar responden mayormente a las trayectorias docentes e institucionales. Dichas trayectorias se circunscriben a los deportes tradicionalmente enseñados en las escuelas y los clubes, con una diferenciación de sus prácticas según el tipo de clasificación sexo-genérica. Esta visión tensiona con los principios del diseño curricular provincial de nivel medio, que delimita los deportes en tanto constructos socioculturales y los organiza desde la valorización de las experiencias lúdicas, la participación equitativa, la comprensión de la multiplicidad de dimensiones intervinientes en su desarrollo, entre otros factores. Las opiniones hacia este documento varían considerablemente, no obstante ello, existen coincidencias con respecto a las dificultades para su análisis y su implementación.

Con este nuevo proyecto nos proponemos analizar las prácticas deportivas desde la perspectiva de género y la teoría de la colonidalidad del poder en nuestra región. Este análisis tiene como objetivo enriquecer los debates de los saberes deportivos a enseñar en educación física, con aportes a la reflexividad acerca de las experiencias, las teorías y las condiciones o 
mecanismos subyacentes. La metodología se configura desde un enfoque cualitativo, el cual atraviesa los diversos componentes de la formulación del proyecto y conlleva una aproximación a la perspectiva de los sujetos, junto a la búsqueda de una objetivación de los discursos y las prácticas. Se incluye además, la elaboración y socialización de narrativas que propicien la mutación de los problemas cotidianos en problematizaciones, y contribuyan a la formulación de propuestas educativas alternativas desde una perspectiva crítica. La reflexividad hacia las prácticas deportivas de interés del estudiantado propicia la apertura escolar a un conjunto diverso y disperso de saberes, y puede accionar en una orientación pedagógica dirigida a revertir los estereotipos de género y raza imperantes en nuestra región.

\section{Perspectiva teórica de la investigación:}

La educación física se ha desarrollado a lo largo de su historia bajo una fuerte dependencia hacia otros campos (Ron, 2003). Desde 1980 esta realidad se ha ido revirtiendo, sobre todo desde las investigaciones efectuadas por los profesorados en educación física con dependencia en ámbitos universitarios. No obstante, todavía existen cuestionamientos en cuanto a las modalidades de articulación que se establecen con las prácticas, a partir de un discurso pedagógico hegemónico que promueve asimetrías entre el saber práctico y el saber teórico, entre investigadores y docentes.

Las prácticas deportivas están regidas por las condiciones específicas de producción del capitalismo global actual y asientan sus bases en la Modernidad. Se encuentran atravesadas por varios procesos, uno de los cuales es la mundialización de las culturas que impone la reorganización del espacio y del tiempo, producto de los impactos de las nuevas tecnologías de la información y la comunicación. En este marco, se lleva a cabo un proceso inédito de amplificación de discursos y prácticas que pugnan por imponerse.

Para el abordaje situado de estas prácticas recurrimos al enfoque interseccional y sus estudios de las relaciones de poder bajo la tríada de opresión "raza-clase-género" (Vigoya, 2016, p. 5). Sus antecedentes se localizan en los feminismos disidentes, en particular el feminismo negro que irrumpe hacia mediados de 1970 en los Estados Unidos. Estas corrientes destacan las diferencias existentes entre las mujeres y llevan a cabo una crítica de las categorías de género y raza al interior de la trama de opresión de clase. El enfoque interseccional comienza a desarrollarse en nuestra región hacia fines del siglo pasado, en sintonía con la crítica de la colonialidad del poder. Sus planteos ponen en evidencia la persistencia de una matriz instaurada desde los tiempos de la conquista, sustentada en la expansión vertiginosa de un "frente colonial/estatal-empresarialmediático-cristiano" (Segato, 2015, p.106). Las categorías de raza y género direccionan las condiciones materiales de existencia, jerarquizan ciertos saberes sobre otros, y se constituyen como los elementos centrales para 
reconstruir los hilos de las experiencias de los pueblos y comunidades intervenidas desde los tiempos de la irrupción europea.

Diversas autoras localizan una similitud en el tratamiento categorial de raza y género, y erigen una crítica respecto de los principios biológicos que legitiman las desigualdades y estabilizan tradiciones racistas, misóginas y homofóbicas (Femenías, 2019; Lugones, 2017; Segato, 2015). Dentro de este pensamiento se observan diferencias entre quienes niegan y quienes afirman la existencia de una organización patriarcal previa a la conquista. Lugones (2017) manifiesta la inexistencia del patriarcado en los pueblos pre-existentes al orden estatal y hace mención a una organización matriarcal y un régimen de deseo sexual que se distancian de los imperativos establecidos con posterioridad. Plantea que el pasaje de comunidades igualitarias y matriarcales a jerárquicas y patriarcales, se desarrolló a la par de la destrucción de los modos de organización tribal y las expulsiones de las tierras, lo que trajo consecuencias en la reconfiguración del trabajo y el reemplazo del clan por la familia nuclear. Asimismo, agrega el accionar funcional de los hombres indígenas dentro del nuevo orden patriarcal, producto de la situación de sometimiento ante los colonos de origen europeo. Por su parte, Segato (2015) expone la existencia de xenofobia y desigualdades de género en el orden preintrusión, plasmadas en dualidades jerárquicas que, a diferencia de los binarismos modernos, mantienen una entidad ontológica y política hacia las diversas posiciones. Esta organización patriarcal difiere del orden colonial en sus aperturas al tránsito y la circulación entre posiciones. La autora menciona como ejemplo que los hombres deliberaban en los espacios públicos de la aldea mientras las mujeres acompañaban con señales de aprobación o desaprobación y los asesoraban luego dentro del espacio doméstico. El patrón colonial y binario se dirigió a desarticular la participación política de las mujeres, afectando las redes de intercambio y las asociaciones internas. Este panorama trajo aparejado un incremento de la vulnerabilidad y del desamparo ante la violencia ejercida por los hombres de sus propios pueblos y comunidades, quienes a su vez padecían el maltrato y la explotación de los europeos en el frente externo.

Estos postulados complejizan los procesos reflexivos de las redes de poder, brindan una visibilidad a las producciones teóricas en América Latina y acompañan las luchas políticas de los sectores postergados a lo largo de la historia. Todo ello involucra directamente a las prácticas deportivas, las cuales actúan sobre los cuerpos a través de técnicas, disposiciones, maniobras, funcionamientos sociales e institucionales, que pueden descifrarse en tanto red de relaciones. En este marco nos preguntamos si la educación física es funcional a este sistema y en qué medida la enseñanza se encuentra atravesada por las categorías de opresión mencionadas. Varios son los interrogantes que nos permiten problematizar ese entramado de relaciones en función de las prácticas deportivas que se desarrollan en las instituciones escolares ¿Por qué se enseñan casi siempre los mismos deportes desde hace décadas en las escuelas secundarias? ¿Cómo se configuran los cuerpos a partir de la selección de dichas prácticas? ¿En qué aspectos se tiene en cuenta la 
diversidad de prácticas de nuestra región? ¿Por qué todavía persisten clases y deportes que se diferencian según una clasificación sexo-genérica?

Los dispositivos de poder se anudan directamente a los cuerpos, en sus funciones, procesos fisiológicos, sensaciones, emociones, constituyendo su propia materialidad. En tal sentido, algunas de las preguntas clave referidas al estudio de los saberes y los sistemas de poder que regulan estas prácticas son: ¿cómo deslindar de una serie de palabras, frases y expresiones, los enunciados y las proposiciones relativas al orden discursivo que las atraviesan? ¿De qué modo el poder performativo del lenguaje habilita o inhibe el acceso a las prácticas deportivas y establece visibilidades e invisibilidades acerca de las experiencias corporales?

Foucault (2003) expone que lo verdadero se presenta a través de las problematizaciones de las prácticas y es allí donde transita el estudio de la historia de la sexualidad como experiencia que conecta las formaciones de saber, los sistemas de poder y los modos en que los sujetos desean y se reconocen a sí mismos. Desde esta perspectiva, toda época o formación histórica pende de una distribución de tipo relacional de lo decible y lo visible (las palabras y las cosas), siendo el saber un agenciamiento práctico de los mismos.

Butler (2002) expresa que el poder performativo del lenguaje oficia como un ideal regulatorio que tiene implicancias materiales y se convierte en un factor fundamental en las representaciones del mundo y las emociones que los sujetos experimentan. Se despliega bajo ciertas condiciones socio-históricas y tiende a la reproducción en virtud de la reiteración forzada de las normas, lo cual es también una señal de su carácter inestable, siempre inconcluso. Si bien resulta imposible situarse subjetivamente por fuera de un discurso, ya que detenta un poder constituyente y subyugante, existe la posibilidad de deliberar acerca del universo simbólico desde su capacidad de resignificación. La localización del sexo y del género como constructos subvierte el heterosexismo y los binarismos dominantes y excluyentes. En tal sentido, y a modo de ejemplo, Butler (2002) recurre al término queer para dar cuenta de esta inversión discursiva, en la medida que deja de ser un estigma paralizante para convertirse en una nueva serie de afirmaciones significativas. Estas afirmaciones visibilizan una pluralidad de modos de sentir y actuar en el mundo, que tensionan al interior del orden discursivo e interpelan la matriz de inteligibilidad hegemónica, desde la producción de nuevas ideologías que permiten "deconstruir" las ya perpetuadas.

Las prácticas deportivas en los diversos contextos forman parte de un orden discursivo que establece visibilidades e invisibilidades, en el marco de las relaciones de poder generadas por las instituciones deportivas, los medios de comunicación, las políticas públicas y el mercado global. Ese nuevo orden discursivo tensiona con las experiencias históricas acumuladas y entrelazadas de los pueblos y las comunidades de nuestra región, obstaculizadas en su devenir por la irrupción colonizadora y patriarcal. El capitalismo ejerce su hegemonía sobre estas trayectorias comunitarias heterogéneas, según diversas formas de trabajo, de explotación y de control, que resultan en parte novedosas si se 
los compara con otras regiones. Todo ello se despliega en instancias de sociabilidad, constituidas en torno a prácticas que históricamente estuvieron bajo dominio masculino, que no se encuentran estrictamente pautadas, y generan sentimientos de pertenencia en función de los objetivos, las normas y los espacios habitualmente compartidos.

Guelman y Palumbo (2018) expresan la necesidad de indagar las "pedagogías descolonizadoras". Entre sus posibles tópicos exhiben una mirada crítica hacia los saberes legitimados al interior de los ámbitos educativos que "(...) exaltan el conocimiento académico y confinan al lugar de subalternidad, carencia e ignorancia a la pluralidad de saberes populares existentes en América Latina" (p. 195). La apertura de la escuela hacia ese conjunto diverso y disperso de saberes corporales favorece el diálogo en las clases de educación física y la enseñanza de los deportes. Este diálogo puede llevarse a cabo desde la interpelación de los sentidos sobrevaluados por las esferas del alto rendimiento y el reconocimiento de valores, aptitudes y emociones muchas veces desestimadas: el placer del juego, los lazos cooperativos y solidarios, la creatividad, la invención, el respeto por la diversidad y la búsqueda del desafío mutuo.

El juego, las emociones y las relaciones intersubjetivas cobran un papel protagónico, en pos de una formación que confronta con las miradas unidimensionales y centralmente cognitivas de los aprendizajes, y recompone lo que la colonialidad del poder y el capitalismo moderno dividieron. Todo ello aporta a una didáctica específica que modifica las reglas de los deportes de acuerdo a las problemáticas e intencionalidades educativas y conserva al mismo tiempo sus lógicas internas dentro de los contextos de juego.

\section{Perspectiva meta-teórica y diseño metodológico:}

Adscribimos a una tarea investigativa que capitalice y a la vez cuestione los esquemas universalistas, que por lo general esconden una visión eurocéntrica y patriarcal en la producción de conocimientos. Las teorías remiten a construcciones lingüísticas que producen diversos significados en torno a los acontecimientos socioculturales y educativos. Las mismas requieren ser comprendidas en el marco de disputas de poder, donde las perspectivas dominantes buscan imponer su visión del mundo con respecto a las subordinadas. Estas pujas se desarrollan en nuestra región en la complejidad de relaciones desplegadas a lo largo del tiempo entre las diferentes naciones y pueblos que componen la herencia económica, política y cultural de la irrupción europea.

Creemos que la matriz socio-histórica y cultural condiciona el planteo del problema, así como también la búsqueda de respuestas, motivo por el cual es menester interpelar los propios desempeños, con el fin de objetivar el universo social y profesional que condiciona las tareas de investigación (Bourdieu, 2003). Asimismo, la política de conocimiento que promulgamos se dirige al establecimiento de una relación simétrica entre trabajo docente e investigación académica. La elaboración de relatos y la problematización de acontecimientos favorecen, en tal sentido, la 
construcción de conocimientos y la elaboración de propuestas para la transformación de la enseñanza de los deportes en las escuelas. Para ello, debemos actuar con oficio en el acompañamiento, la apertura hacia nuevas técnicas de construcción de datos, en los aportes de nueva bibliografía y las interpretaciones que reclama toda tarea investigativa. De igual modo, consideramos de vital importancia la elaboración de textos que incorporen diálogos puestos a consideración con quiénes participan directamente en la investigación y habiliten una variedad de lecturas e interpretaciones posibles.

Las prácticas de investigación acción basadas en la reflexividad y en el diálogo de saberes orientan una pedagogía descolonizadora en clave de género, con una teoría de la práctica que reconstruye los acontecimientos en sus atravesamientos socioculturales y formula categorías comprometidas con los sectores sociales vulnerados. Todo ello contribuye a la configuración de una posición epistémica activa y participativa, en la medida que la comunidad docente produce conocimientos desde las prácticas, y la comunidad estudiantil enseña saberes construidos en sus contextos cotidianos.

La reflexión sobre la acción opera como eslabón, como punto de encuentro entre la teoría y la práctica, se activa en las pausas que se establecen dentro o entre una clase y otra, y a partir de ella es factible revisar los propósitos y los fundamentos que guían el sentido práctico. Se interpela así la reflexión dada en la acción, a partir de los conceptos, las teorías, los saberes que circulan y en nuestro caso, al interior del campo de la educación física. Es interpretativa porque resulta imperioso otorgar sentido a las prácticas, fruto de los debates colegiados, la construcción de datos y las teorías que le dan sustento, y abarca diversos niveles de análisis que podríamos situarlos en las experiencias, los acontecimientos y los mecanismos o estructuras subyacentes.

El proyecto se enmarca dentro de un desarrollo metodológico de tipo cualitativo y en tanto proceso sistemático de interpretación no pretende verificar teorías. Implica un trabajo intelectual de análisis de datos, que procura contribuir a los conocimientos disponibles sobre las prácticas deportivas comunitarias con participación juvenil y a los procesos de selección de saberes a enseñar en educación física de las ESRN de San Carlos de Bariloche. Las interpretaciones se sustentarán en la construcción de datos y en los procesos de reflexividad, los cuales conllevan una diferenciación e interacción entre las significaciones del equipo de investigación y las significaciones de los sujetos implicados a lo largo del trabajo. Las teorías y las metodologías se irán vinculando de forma dinámica a las evidencias discontinuas, incompletas y parciales de las prácticas, en sus enlaces con los condicionamientos socioculturales, políticos y económicos. Desde aquí los datos serán el resultado de construcciones interactivas y dialógicas, dentro de un proceso simultáneo e indisociable de observación, participación, registro e interpretación dentro del mismo trabajo de campo.

El estudio se compone en torno a un diseño no experimental de tipo descriptivo, realizado mediante estudio transversal, que implica un 
análisis en un corte temporal específico. El criterio de selección de las instituciones escolares se sustenta en el alcance territorial y el tipo de articulación que las mismas establecen con las comunidades barriales. La unidad de análisis se compone en torno a los discursos y las prácticas de visibilidades en los eventos deportivos comunitarios con participación juvenil, en sus articulaciones con los procesos de selección de saberes a enseñar en educación física de las ESRN de San Carlos de Bariloche.

A lo largo de la investigación haremos una triangulación de los datos construidos a partir de las entrevistas en profundidad, las observaciones, el análisis de documentos y la elaboración de narrativas, con el fin de suplir las carencias que cada una de estas técnicas presenta de forma aislada. La elaboración de narrativas tienen como objetivo reconstruir y hacer públicos los acontecimientos comunitarios y educativos cotidianos, en textos que exhiben formas particulares de pensar y actuar dentro de ellos. Tarea nada sencilla si tenemos en cuenta los requerimientos de este tipo de dispositivo, que consiste en la generación de condiciones institucionales para su puesta en práctica, que plantea el involucramiento subjetivo con escritos narrados en primera persona y demanda versiones sucesivas de relatos fruto de los intercambios, con requisitos de capacitación para su publicación y difusión (Suárez, 2012). Los documentos narrativos no se producen de manera espontánea, por el contrario, son consecuencia de un proceso complejo y laborioso que demanda la participación activa de todos los sujetos implicados. Posibilitan desandar los sentidos desapercibidos o ignorados y las formas de relación intersubjetiva que se establecen dentro del mundo escolar y comunitario. Por todo esto, la problemática de la textualización cobra vital interés ya que, si bien al momento de la escritura resulta muy difícil escapar del estilo indirecto, de los recortes de las enunciaciones y las traducciones a un formato que detente cierta lógica y coherencia según los estándares académicos; es factible pensar en ciertos controles. Entre ellos, la elaboración de textos que incorporen diálogos, que permitan una variedad de lecturas e interpretaciones posibles de las experiencias indagadas, donde la socialización de los mismos se lleve a cabo con quienes colaboran directamente en la investigación. Textos que en definitiva propendan a ser un "(...) diálogo ficcional (...) una representación simplificada de complejos procesos multívocos” (Clifford, 1998, p. 166).

Los siguientes conceptos de estudio orientarán el análisis de las prácticas deportivas comunitarias con participación juvenil: trayectorias histórico/estructurales, sociabilidad, saberes corporales, juego, orden discursivo y prácticas de visibilidades de género-raza-clase. Asimismo, los conceptos que mencionamos a continuación orientarán los procesos de reflexividad respecto de la selección de saberes deportivos a enseñar: saberes corporales estudiantiles, diseño curricular para las ESRN, juego, categorías de opresión de género-raza-clase. 


\section{Resultados previstos y breves consideraciones finales:}

Con el nuevo proyecto de investigación nos proponemos favorecer una pedagogía descolonizadora en clave de género, con una mirada crítica hacia las prácticas docentes y una comunidad estudiantil que enseña y problematiza los saberes construidos en los contextos cotidianos. Este ideal discrepa con el esquema didáctico lineal que se legitima exclusivamente en la cercanía con el campo científicoacadémico y que dictamina un saber aprendido referenciado en el estudiantado, último eslabón de la cadena de mediaciones. De igual modo, pretendemos que el estudio aporte al cuestionamiento de la concepción de conocimiento como elemento externo, que metafóricamente se vierte sobre los sujetos inmersos en la trama educativa. Los intercambios de saberes que en ella se establecen son producto de trayectorias subjetivas/socioculturales, materializadas en los cuerpos de docentes y estudiantes. Saberes que se actualizan en situaciones concretas de las prácticas y pueden utilizarse como herramientas para entender y actuar con inventiva en los diversos ámbitos y contextos.

La validación y problematización de las prácticas deportivas que la comunidad estudiantil desarrolla de forma cotidiana, se inscribe en las luchas políticas por los derechos de género, de los pueblos pre-existentes al orden estatal vigente y los diversos sectores vulnerados de nuestra sociedad. Los aportes al conocimiento de este trabajo se orientan a un "trabajo político de representación" en pos de elevar "a la objetividad del discurso público (...) una manera de ver y de vivir el mundo relegada al estado de disposición práctica o de experiencia tácita y a menudo confusa" (Bourdieu, 1985, p. 98). Todo ello puede habilitar la construcción de alianzas corporales que atraviesen la diversidad de experiencias y acontecimientos. Alianzas que establezcan disputas políticas y reconfiguraciones de las categorías hegemónicas subyacentes. Se trata en definitiva de interpelar y apelar al poder performativo del discurso, y así brindar la posibilidad de reconsiderar lo simbólico desde su capacidad de resignificación. Desde aquí consideramos factible construir un reconocimiento que habilite a las poblaciones vulneradas o precarizadas a hacer visibles sus reivindicaciones, tales como, el derecho a recibir el apoyo social e institucional necesario para su desarrollo.

Creemos que desde el área de la educación física es posible favorecer la "política de alianza" de las diversas poblaciones precarizadas dentro de la esfera educativa (Butler, 2017, p. 75). Desde esta perspectiva, la enseñanza de los deportes emprendería una lucha generalizada contra la precariedad, haciendo visible las relaciones de interdependencia y el sentido político de los cuerpos. Asimismo, estaría operando en el plano de las condiciones infraestructurales y ambientales, las cuales actúan como soportes materiales toda vez que se lucha por ellas. 


\section{Referencias bibliográficas:}

Butler, J. (2017). Cuerpos aliados y lucha politica. Hacia una teoría performativa de la asamblea. Buenos Aires: PAIDÓS.

Butler, J. (2002). Cuerpos que importan. Sobre los limites materiales y discursivos del "sexo". Buenos Aires: Paidós.

Bourdieu, P. (2003). La objetivación participante. Traducido del francés por Paula Miguel. L'objectivation participante. Actes de la rechenhe en sciences sociales, No 150, (43-58). Recuperado de: https://campus.fahce.unlp.edu. $\mathrm{ar} / \mathrm{mod} /$ resource $/$ view.php?id $=448855$

Bourdieu, P. (1985) ¿Qué significa hablar? Economia de los intercambios lingüisticos. Madrid: Akal.

Clifford, J. (1991/1998). La autoridad etnográfica. En Geertz y Clifford (Eds.). El surgimiento de la antropología posmoderna (pp.141-170). Barcelona: Gedisa

Femenías, M. (2019). Itinerarios de la teoría feminista y de género. Algunas cuestiones histórico-conceptuales. Universidad Nacional de Quilmes. Recuperado de: http://www.unq.edu.ar/advf/documentos/5cf00faf7c05 d.pdf

Foucault, M. (1984/2003). Historia de la sexualidad. 2. El uso de los placeres. Buenos Aires: siglo veintiuno editores.

Guelman y Palumbo (2018). Pedagogías descolonizadoras. Formación en el trabajo en los movimientos populares. Buenos Aires: El Colectivo, CLACSO.

Lugones, M. (2017). Colonialidad y género: hacia un feminismo descolonial. En Mignolo (Ed.). Género y descolonialidad(pp. 13-42). Buenos Aires: Ediciones del signo.

Martins, Fabián H. (septiembre-octubre, 2019) ¿Qué hacemos con los deportes en las escuelas secundarias rionegrinas? Trabajo presentado en 13 Congreso Argentino de Educación Física y Ciencias. Universidad Nacional de La Plata. La Plata. Resumen recuperado de: http://www.memoria.fahce.unl p.edu.ar/trab_eventos/ev.12777/ev.12777.pdf

Ron, O. (2003). El Campo de la Educación Física. En Bracht y Crisorio (Eds.). La Educación Física en Argentina y Brasil. Identidad, desafios, perspectivas (pp. 59-73). La Plata: Al Margen.

Segato, R. (2015). La critica de la colonialidad en ocho ensayos y una antropología por demanda. Buenos Aires: Prometeo.

Suárez, D. (2012). Docentes, narrativa e investigación educativa. La documentación narrativa de las prácticas docentes y la indagación pedagógica del mundo y las experiencias escolares. En Sverdlick (Ed.). La investigación educativa. Una herramienta de conocimiento y de acción (pp. 71-110). Buenos Aires: Novedades Educativas.

Vigoya, M. (2016). La interseccionalidad: una aproximación situada a la dominación. Revista Debate Feminista N ${ }^{\circ}$ 52, (1-17). Recuperado de: http://www.debatefeminista.cieg.unam.mx/wp-content/uploads/20 16/12/articulos/052_completo.pdf 


\section{Notas}

1 Proyecto de investigación denominado "Sociabilidad, deporte y educación física: análisis para la enseñanza situada de los saberes de corporales en las escuelas secundarias. San Carlos de Bariloche, provincia de Río Negro, período 2021-2024". Profesorado en educación física de la Universidad Nacional del Comahue, sede Bariloche. Director: Mg. Fabián Martins. Codirector: Lic. Federico Pizzorno. Fecha estimada de presentación a la Secretaría de Ciencia y Técnica: marzo de 2020.

2 Proyecto de investigación denominado "Contenidos y enseñanza de los deportes en educación física. Ciclo básico de nivel medio de San Carlos de Bariloche, período 2017-2020" (04/B210), perteneciente al departamento de educación física de la Universidad Nacional del Comahue, sede Bariloche. Director: Mg. Fabián Martins. Codirector: Lic. Federico Pizzorno. 